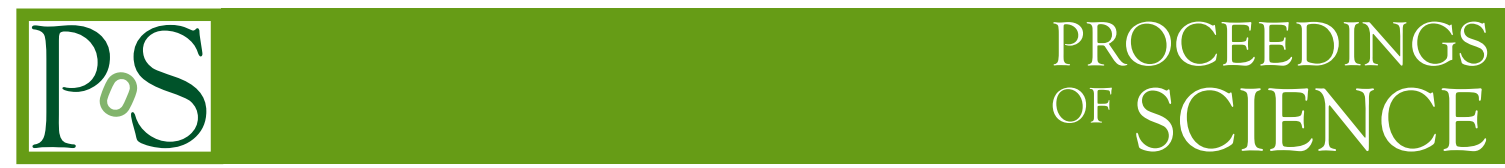

\title{
Prospects on Muon Colliders
}

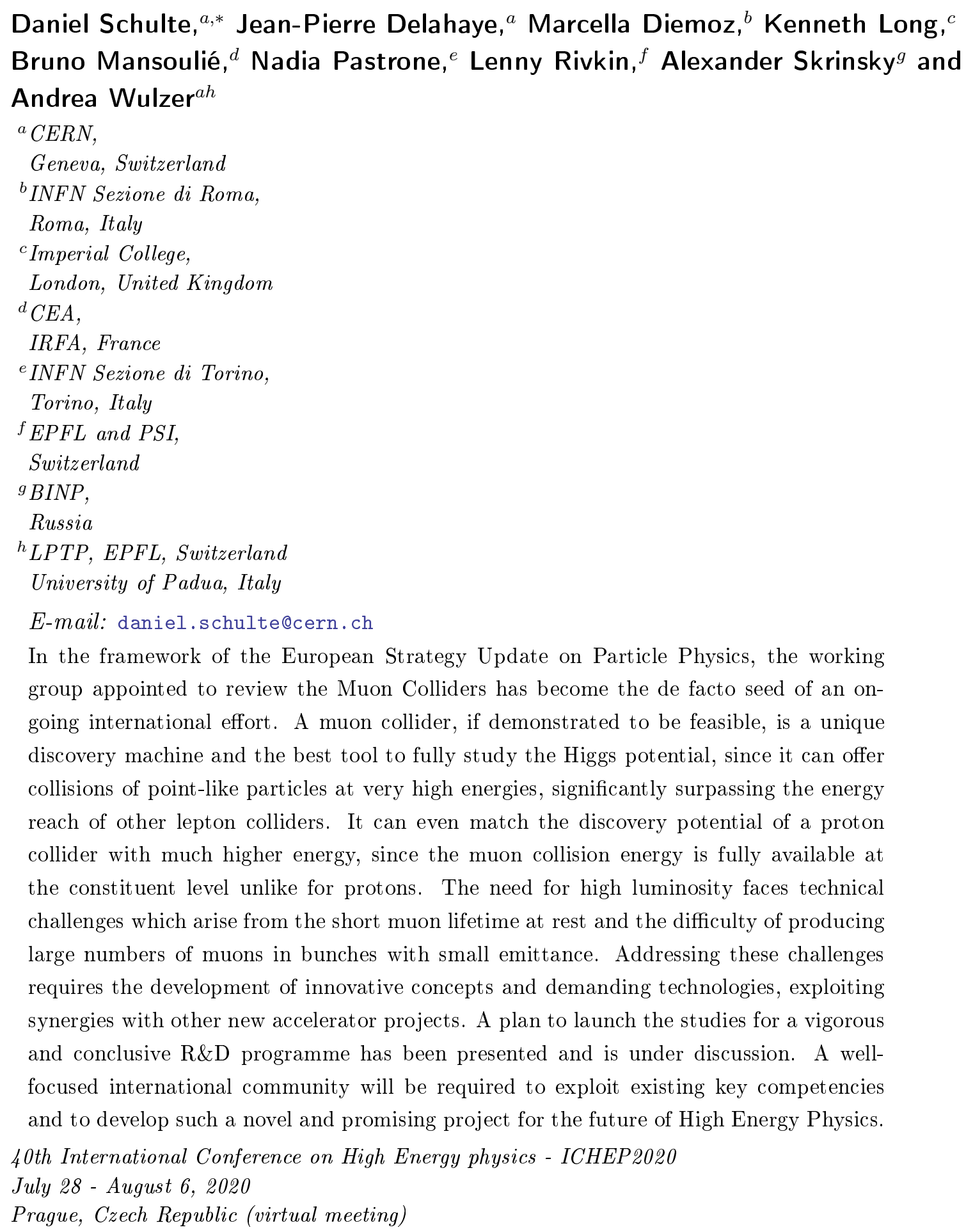

${ }^{*}$ Speaker 


\section{Motivation}

Circular muon colliders have the potential to reach centre-of-mass energies of tens of $\mathrm{TeV}$ with high luminosity[1]. Muons are point-like particles, so the entire nominal energy is available to produce short-distance reactions, which allows direct searches for new particles in particular to be carried out over a wide range of unexplored masses. A muon collider also allows accurate tests of the Standard Model to be performed at extremely high energy, offering great opportunities to detect new physics indirectly and/or to confirm and to characterise direct discoveries. Furthermore, by exploiting the copious rate for Vector Boson Fusion and Vector Boson Scattering processes, the muon collider provides the opportunity to probe the most intimate nature of the Electroweak Symmetry Breaking mechanism.

The MAP collaboration[2] has developed the muon collider concept and technologies; the efforts have been reduced several years ago. The concept is based on a proton-driven muon source and requires muon beam cooling, which has been tested in the UK by the MICE collaboration[3]; results were recently published. The LEMMA team[4] has proposed to use positrons for muon beam production, which would not require cooling. With innovations this could become an important alternative solution and simplifiy a number of design issues.

The European Strategy for Particle Physics therefore recommended to form a new international muon collider study as a part of the accelerator R\&D roadmap[5]. In Europe, the European Large National Laboratories Directors Group (LDG) has subsequently initiated an international muon collider collaboration[6], which covers the physics, detector and collider facility. The work has started[7].

\section{Scope}

The study aims to establish whether a muon collider is feasible and, if so, to develop the concept and technology to a level of maturity that allows committing to its construction supported by a validation of the physics reach. This full conceptual design is expected to demand a very important effort, similar to other large projects. In particular, the technology challenges motivate further $\mathrm{R} \& \mathrm{D}$, prototype construction and performance demonstrations.

The study will focus on high energies, in particular $3 \mathrm{TeV}$ and $10 \mathrm{TeV}$ or more. The tentative goal for the integrated luminosity target, which will be refined later, would be

$$
\int \mathcal{L}=10 \mathrm{ab}^{-1}\left(\frac{\sqrt{s}}{10 \mathrm{TeV}}\right)^{2}
$$

The $3 \mathrm{TeV}$ case, with a luminosity of $\mathcal{L} \approx O\left(10^{34} \mathrm{~cm}^{-2} \mathrm{~s}^{-1}\right)$, would substantially extend the energy reach of superconducting linear colliders and allows the study to benefit from the work on the machine that has been performed in the US and elsewhere as well as from the physics and detector studies performed for CLIC.

The case of $10 \mathrm{TeV}$ or more, with a luminosity of the order of $\mathcal{L} \approx O\left(10^{35} \mathrm{~cm}^{-2} \mathrm{~s}^{-1}\right)$, would extend lepton collider to an energy range inaccessible even to normal conducting linear colliders. A muon collider operating in this energy range will open entirely new physics opportunities in the domains of Higgs/Electroweak and Beyond the SM physics. 


\section{Overall Plan}

A muon collider, in particular at an energy of $10 \mathrm{TeV}$ or above, is uncharted teritory. Therefore the study has to explore the full physics reach and develop a concept of the detector that can realise the physics potential and mitigate background effects. An integrated collider concept has to be devised that can deliver the required luminosity and background conditions. It has to have acceptable impact on the environnement and be cost and power efficient. The study will profit from the substantial progress has been made in the design of the muon collider and in the development of its technologies[1, 2, 4].

In the first period, in time for the next European Strategy for Particle Physics Update, the study aims to establish whether the investment into a full CDR is justified. It will provide a baseline concept, well-supported performance expectations and assess the associated key risk as well as cost and power consumption drivers. It will also identify an R\&D path toward a full CDR for the machine and the associated experiments.

Depending on the strategic decision, this could be followed by a period of about six years to develop an optimised conceptual collider design with cost and power consumption estimate. This period focuses on the construction and operation of key prototypes for performance validation and on the construction and operation of the required test facility infrastructre to demonstrate the concept. The conceptual design will be the technical basis for a decision on whether to commit to the project. In the this case a technical design can be developed over a period of about four years. This would require a continuation of the technology development and more integrated tests with beam.

\section{Initial Workplan}

The work until the next European Stratgy Update will be split in two phases, the exploratory phase, which will last about two years, and the definition phase.

In the exploratory phase, the collaboration will define goals for the muon collider performances and develop a prioritised list of key challenges that have to be addressed in the definition phase. The physics reach and its dependence on luminosity and detector performances will be studied. The potential detector performances with existing and novel technologies will be established and the potential impact of background will be considered. The potential performance of the machine will be explored. Based on these initial studies, ambitious and achievable goals will be defined for the definition phase.

Associated to the goals, a list of key challenges to achieve them will be identified. Its prioritisation will serve as the basis to define the work programme in the definition phase. Starting from a tentative list, an initial list of key issues is expected to be ready in time for the Snowmass Process and a refined one at the end of the exploratory phase. The exploration of the detector and the whole collider complex, the development of key concepts and the definition of critical parameters will be essential to identify issues and in particular to prioritise them.

In the definition phase, the workprogrammes developed in the exploratory phase will be implemented for the physics, experiment and facility and might require an increase in 


\begin{tabular}{|c|c|c|c|c|c|}
\hline Parameter & Symbol & unit & & & \\
\hline Centre-of-mass energy & $E_{c m}$ & $\mathrm{TeV}$ & 3 & 10 & 14 \\
Luminosity & $\mathcal{L}$ & $10^{35} \mathrm{~cm}^{-2} \mathrm{~s}^{-2}$ & 1.8 & 20 & 40 \\
Collider circumference & $C_{\text {coll }}$ & $\mathrm{km}$ & 4.5 & 10 & 14 \\
\hline Muons/bunch & $N$ & $10^{12}$ & 2.2 & 1.8 & 1.8 \\
Repetition rate & $f_{r}$ & $\mathrm{~Hz}$ & 5 & 5 & 5 \\
Beam power & $P_{\text {coll }}$ & $\mathrm{MW}$ & 5.3 & 14.4 & 20 \\
Longitudinal emittance & $\epsilon_{L}$ & $\mathrm{MeVm}$ & 7.5 & 7.5 & 7.5 \\
Transverse emittance & $\epsilon$ & $\mu \mathrm{m}$ & 25 & 25 & 25 \\
\hline IP bunch length & $\sigma_{z}$ & $\mathrm{~mm}$ & 5 & 1.5 & 1.07 \\
IP betafunction & $\beta$ & $\mathrm{mm}$ & 5 & 1.5 & 1.07 \\
IP beam size & $\sigma$ & $\mu \mathrm{m}$ & 3 & 0.9 & 0.63 \\
\hline
\end{tabular}

Table 1: Tentative target parameters for a muon collider at different energies based on the MAP design. These values are only to give a first, rough indication. The study will develop coherent parameter sets of its own.

resources as more detailed studies might be needed. The effort will consist of studies to support the ability of reaching the performance goals and to refine these goals where required. Identification and conceptual design of the key demonstration facilities and components will define the R\&D path for the CDR phase and allow to assess its cost and timeline. The construction of models for key hardware might be required during this phase.

Key issues that will be addressed are:

- The impact of muon decays on the experiment, the machine and the environment. Methods to mitigate this impact will be developed. These include civil engineering, shielding, optics and magnet design.

- The ability to efficiently accelerate the beam, to preserve its quality and to obtain the luminosity. This requires development of efficient magnets and power converters, $\mathrm{RF}$ that can accelerate the short intense bunches and maintain their quality as well as shielding and cooling development in combination with optics design to minimise the loss induced heat load.

- The production of a high-quality beam. This includes the proton target and its surrounding and the cooling; the MAP design will be reviewed and optimised also using achieved hardware performances. In particular the final cooling stage has to be improved with higher field magnets.

- Development of the positron-based muon source as an alternative.

Based on scaling from MAP parameters, initial targets for the integrated luminosities have been defined, namely 1,10 and $20 \mathrm{ab}^{-1}$ for 3,10 and $14 \mathrm{TeV}$, see table 1 . These parameters will help to identify the key issues and have to be refined to include findings of 
the studies, e.g. budgets for the emittance growth between the source and the collider ring. A similar list of parameters for a collider using the positron-based muon sources remains to be done once this concept is sufficiently advanced.

\section{Key R\&D}

The production of bright muon beam is instrumental for a collider. The more developed and studied proton-driven option still requires a final demonstrator and further optimisation. The appealing positron driven source, at present missing a feasible design to produce high luminosity, demands dedicated R\&D studies and tests also in synergy with other ongoing projects.

The high-energy acceleration complex and the collider ring contain important performance, cost and power consumption challenges that can ultimately define the energy reach of the collider. A wide range of cutting edge technologies, detailed studies and R\&D is required. In particular:

- Advanced accelerator design and beam dynamics for high luminosity and power efficiency.

- Robust targets and shielding for muon production and cooling as well as collider and detector component shielding and possibly beam collimation.

- High field, robust and cost-effective superconducting magnets for the muon production, cooling, acceleration and collision. High-temperature super-conductors would be an ideal option.

- High-gradient and robust normal-conducting RF to minimise muon losses during cooling.

- High rate positron production source and high current positron ring.

- Fast ramping normal-conducting, superferric or superconducting magnets that can be used in a rapid cycling synchrotron to accelerate the muons.

- Efficient, high-gradient superconducting RF to minimise power consumption and muon losses during acceleration.

- Efficient cryogenics systems to minimise the power consumption of the superconducting components and minimise the impact of beam losses.

- Other accelerator technologies including high-performance, compact vacuum systems to minimise magnet aperture and cost as well as fast, robust, high-resolution instrumentation.

The goal of the collaboration will be to address these challenges.

Initially, the design of the collider will focus on a green field option that allows to fully focus on technical issues. Once the design and understanding of the concept has evolved sufficiently, the option to reuse existing infrastructures will also be explored. 


\section{References}

[1] J. P. Delahaye, M. Diemoz, K. Long, B. Mansoulié, N. Pastrone, L. Rivkin, D. Schulte, A. Skrinsky, and A. Wulzer, "Muon Colliders", arXiv:1901.06150.

[2] https://map.fnal.gov/

[3] Bogomilov, M., Tsenov, R., Vankova-Kirilova, G. et al. "Demonstration of cooling by the Muon Ionization Cooling Experiment", Nature 578, 53-59 (2020). https://doi.org/10.1038/s41586-020-1958-9

[4] LEMMA Team, "Positron driven muon source for a muon collider", arXiv:1905.05747v2 [physics.acc-ph]

[5] The European Strategy Group,"Deliberation document on the 2020 Update of the European Strategy for Particle Physics", CERN-ESU-014, 2020, https://cds.cern.ch/record/2720131.

[6] European Large National Laboratories Directors Group (LDG), Minutes of the LDG meeting on July 2, 2020.

[7] http://muoncollider.web.cern.ch. First meeting https://indico.cern.ch/event/930508/, 272 participants. 